\title{
Study on level-of-service for urban rail transit passages from the passenger perspective
}

\author{
Yibing Chen ${ }^{1}$, Xiao Liang ${ }^{1 *}$, and Yizhuo Fu ${ }^{3}$ \\ ${ }^{1,3}$ School of traffic and transportation, Beijing Jiaotong University, Beijing
}

\begin{abstract}
With the acceleration of urbanization and the shortage of transportation resources in large cities, urban rail transit has gradually become the backbone of urban public transportation system. In order to improve the service level of urban rail transit, this paper studies the factors influencing the service level of subway station passages and the classification method based on the passenger-perception method. Through investigation and research, the passage service level evaluation indicators (width, walking time, per capita area, illumination intensity and guide signage) have been determined. Based on orthogonal experiment, $3 \mathrm{ds}$ max was used to set up 25 passage scenes with different parameters from passengers' perspective. A passenger satisfaction questionnaire was designed and distributed, the reliability and validity of the questionnaire were tested to ensure the validity of the data. And based on the factor analysis method, the factors affecting passenger perception are analyzed. A well-fitting model of the relationship between passenger scores and passage parameters is established, and the passenger scores are converted into service levels. Finally, a service level classification method is given, which can provide reference for the service level evaluation of existing stations and the design of physical attributes and environmental factors of new station passages.
\end{abstract}

\section{Introduction}

With the development of China's urban rail transit network, improving passenger service levels has become an important goal of urban rail transit planning and operation. As an important facility for passenger collection and transfer, the passage in the station is a key point that restricts the service level. This paper will study the classification method of passage service level in line with passengers' perception, and provide reference for the service level evaluation standard setting of existing stations and the design of new station passages.

The current passage service level evaluation methods include experts' subjective perception evaluation, fuzzy evaluation, and passenger-perception evaluation. Mumayiz $^{[1]}$, Anderson ${ }^{[2]}$, Shouhua $\mathrm{Cao}^{[3]}$ and others conducted researches based on passenger-perception, and had many research results in impact indicators analysis and service level threshold division, but the following problems still exist. First, commonly used pedestrian service level standards such as Highway Capacity Manual ${ }^{[4]}$ and Transit Capacity and Quality of Service Manual ${ }^{[5]}$ do not fit the actual needs of subway passengers. Second, there is a lack of industry uniform standards, and there are differences in classification basis and threshold determination. Third, related studies mostly use a single evaluation index. Fourth, the passage scenes cannot be set according to the specific parameters, the actual passage pictures cannot be used to study the impact of channel attribute design on service level.
This study based on passenger-perception methods, obtaining factors affecting passengers on facility satisfaction perception, establishing the relationship between passenger satisfaction and transfer passage design, and connecting passenger satisfaction with service level by establishing a scoring model, Thereby, a passage service level classification method is obtained. It is designed to achieve the purpose of improving the level of transportation of rail transit in the facility design. Aims to improve the design of the passage facility to achieve the purpose of improving the service levels.

\section{Passage service level experiment}

\subsection{Service level evaluation index design}

The service level evaluation indicators based on passenger-perception are related to the influencing factors of the passengers to perceive the service level in the passages.

This study conducted a total of 29 subway transfer stations on the line 2, 4, 10. And combined with relevant literatures, five factors affecting subway passage service levels are summarized in both physical facilities and passenger physiological and psychology. And clarify the corresponding evaluation indicators as width, walking time, per capita area, illumination intensity, and guide signage. The five indicators are graded according to the existing standards and literatures, and the results are shown in Table 1.

\footnotetext{
*Corresponding author: liangx@bjtu.edu.cn
} 
Table 1. Passage service level evaluation indictors and grading

\begin{tabular}{|c|c|c|c|}
\hline & Factor & Indictor & Grading level \\
\hline $\mathrm{A}$ & Passage openness & Width/m & $3,4,5,6,7$ \\
\hline B & Passage length & Walking time/s & $25,45,60,75,90$ \\
\hline $\mathrm{C}$ & Crowdedness & per capita area $/ \mathrm{m}^{2}$ & $1,2.5,5,7.5$ \\
\hline $\mathrm{D}$ & Lighting conditions & $\begin{array}{l}\text { Illumination } \\
\text { intensity/lux }\end{array}$ & $100,150,200$ \\
\hline $\mathrm{E}$ & $\begin{array}{l}\text { Clarity and continuity } \\
\text { of guiding information }\end{array}$ & Guide signage & $\begin{array}{l}\text { Strip signage, } \\
\text { signboard }\end{array}$ \\
\hline
\end{tabular}

\subsection{Passage scenes design based on orthogonal experiment}

According to Table 1, the mixed orthogonal experiment is designed, the number of passage scenes should be set to $25\left(\mathrm{~L}^{25}: 5^{2} \times 4^{1} \times 3^{1} \times 2^{1}\right)$.

Use SPSS to output specific parameters of 25 passages, which sets the horizontal combination of the indicators that each passage should be employed in the subsequent
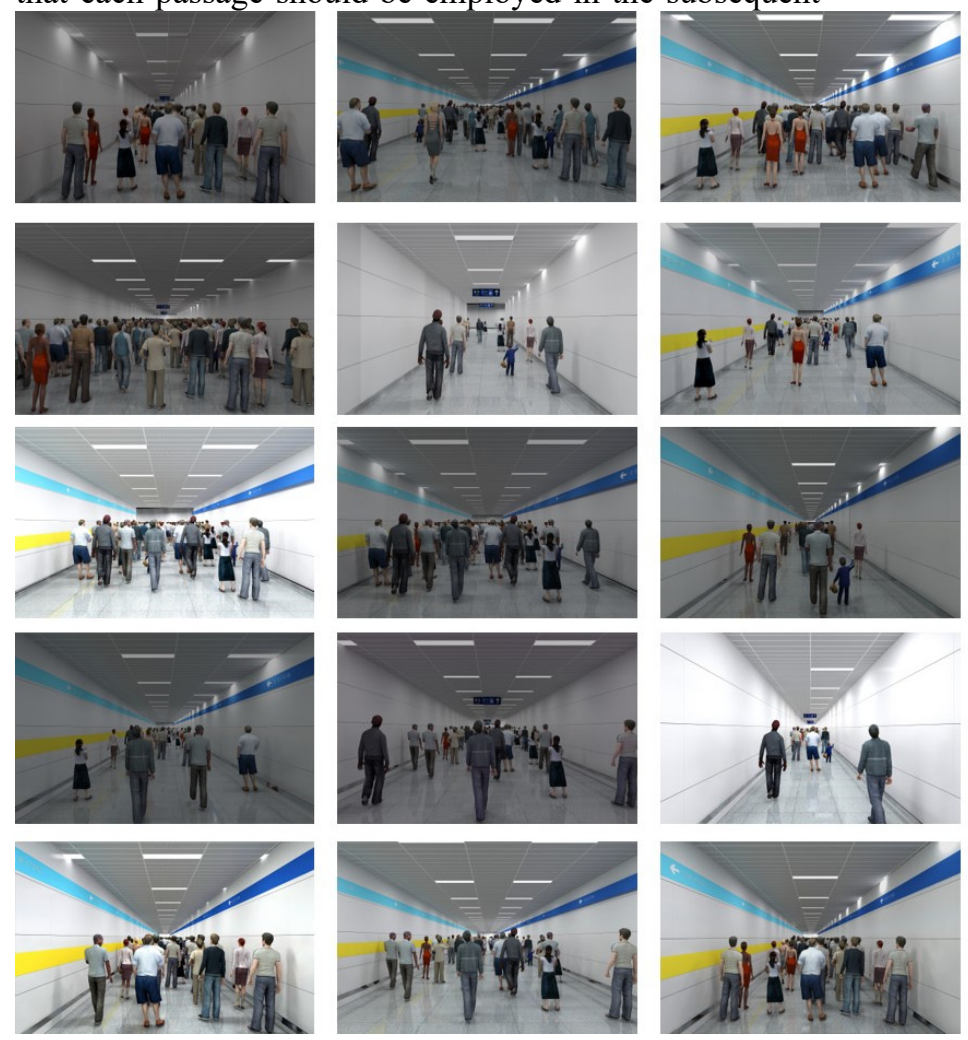
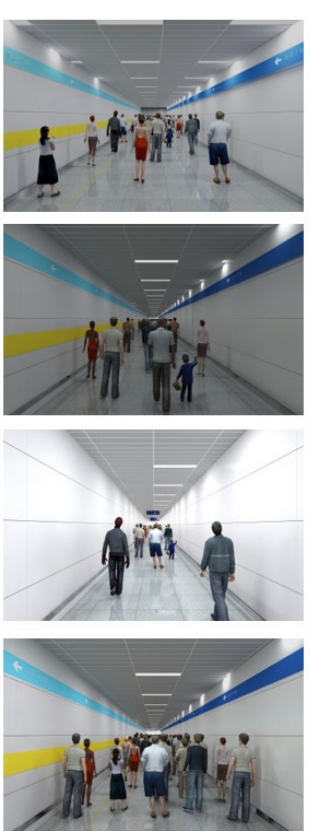

passage service level experiment.

\subsection{D passage scenes modeling}

The experimental scene modeling is a key part of this study. In order to enable passengers with an immersion experience when scoring, improve data reliability, this study uses $3 \mathrm{ds}$ max to create real photo-level experiment scenes, and establish 25 channel scenes in accordance with the passages parameter design results.

First, the passage physical structure (width, length, and guide signage) is modeled. Second, material parameters such as illumination intensity are set. Then set up 25 groups of different quantities and density passenger populations, respectively import the model. Finally, select the first perspective of the passenger walking in the channel, and the model is rendered one by one, and finally the experiment scenes is shown in Fig. 1.
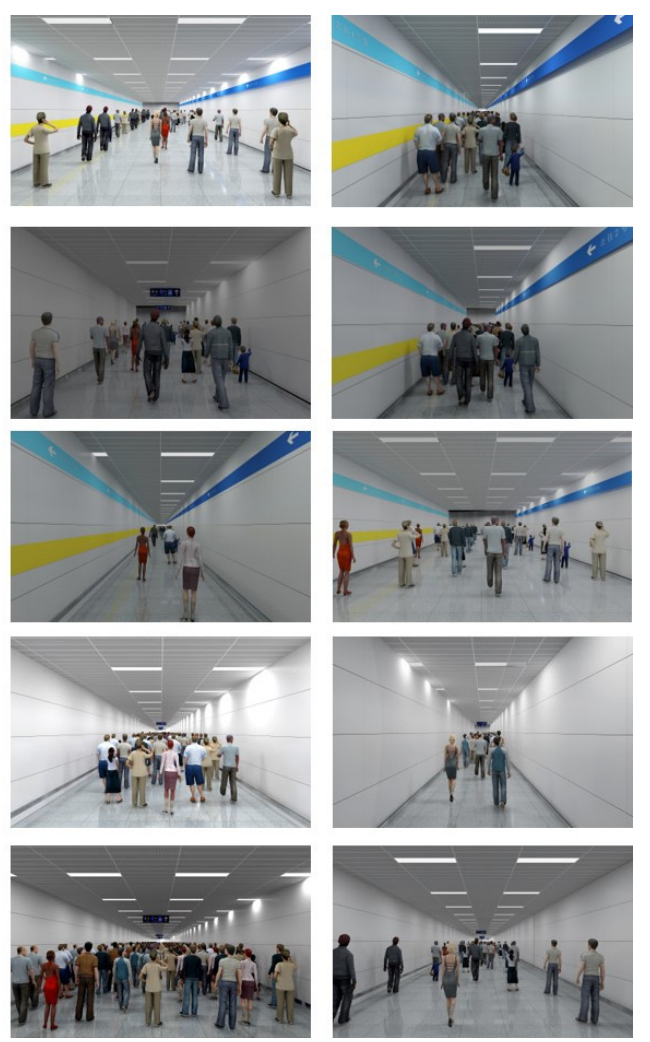

Fig. 1. Passage experiment scenes

\subsection{Service level questionnaire design}

The passage service level questionnaire issued in this experiment mainly includes three parts: guiding language, passenger attribute topics and service level sensation topics. Since the questionnaire design will affect the data reliability, it is necessary to further carry out reliability and validity analysis.

\subsubsection{Questionnaire topic design}

In order to collect the basic composition data of the subjects and ensure the diversification of passenger attributes, the questionnaire has three questions about the basic attributes of passengers, including gender, age, and travel purpose.

The main body of the questionnaire includes 25 service level perception questions, which allow participants to score different passage scenes from the first perspective. Each question consists of a description of the service level experience and a picture of the passage scene under different factors and levels. Using the Likert method, the passenger score is set at 1-5 points. The higher the score, the more satisfied the passengers are with the passage. 


\subsubsection{Reliability and validity analysis}

Due to the design of the questionnaire, the consistency and correctness of the survey results will affect the credibility and accuracy of the data. Therefore, it is necessary to analyze the reliability and validity of the questionnaire.

Use the $\alpha$ reliability coefficient (ie cronbach's $\alpha$ coeffient) to test the reliability of the passenger-perception question, $\alpha \in(0,1)$. The test result shows that $\alpha$ is 0.935 (the reliability of the questionnaire is good when $\alpha \geqslant 0.9$ ). Next, factor analysis was used to test the structural validity of the questionnaire, and the KMO value was 0.927 (it is generally believed that the structural validity is good when the KMO value is greater than 0.7). The results of the questionnaire are representative of the characteristics of the survey content, and the measured data can be used for subsequent analysis.

\section{Data analysis of experimental results}

The questionnaire was distributed through social media. After screening out the invalid data with too short answer time and the same score in each scene, 474 valid questionnaires were collected, that is, each factor contains 11,850 pieces of data (a total of 25 experimental scenes), and the amount of experimental data is sufficient.

\subsection{Participant attribute analysis}

The passengers participating in the survey came from many regions, and the ratio of men to women was roughly equal ( $40.51 \%$ male and $59.49 \%$ female). Covering a wide range of age groups, the largest age group is 16-25 years old $(65.19 \%)$, followed by $25-35$ years old $(16.46 \%)$. The main purpose of the surveyed passengers is leisure travel $(60.34 \%)$, followed by commuting (28.06\%) and commuting to school $(7.39 \%)$.

\subsection{Factor effect analysis}

In order to eliminate the influence of experimental errors caused by accidental factors, variance analysis was carried out on the data, and the results showed that under $95 \%$ confidence level, the significance levels of the four indicators except the guide signage were far less than $5 \%$, which had a significant impact on the channel service level score. As the sample size is sufficient, factor effect analysis is carried out on the basis of mean value to explore the influence mechanism of passage facilities design on service level.

\subsubsection{Passage width}

From the data in Table 2, it can be seen that the score increases with the width, but the growth rate of the score between each adjacent interval decreases continuously, and the decline shows an increasing trend. This shows that passengers can get a better walking experience in a wider passage, but after the width increases to a certain value, the passengers' sensitivity to width increase will decrease. Therefore, it is not necessary to increase the width too much to improve the service level when designing the passage, and the dual requirements of service level and construction cost can be met within a reasonable width range.

Table 2. Descriptive statistics of passage width score

\begin{tabular}{|c|c|c|c|c|c|c|}
\hline \multicolumn{2}{|c|}{ Width/m } & 3 & 4 & 5 & 6 & 7 \\
\hline \multicolumn{2}{|c|}{ Mean score } & 2.27 & 2.58 & 2.74 & 2.73 & 2.83 \\
\hline \multirow{2}{*}{$\begin{array}{l}\text { Confidence - } \\
\text { interval }(95 \%)\end{array}$} & Upper limit & 2.31 & 2.63 & 2.78 & 2.77 & 2.89 \\
\hline & Lower limit & 2.22 & 2.53 & 2.69 & 2.68 & 2.77 \\
\hline \multicolumn{2}{|c|}{ Growth rate of score } & - & $13.66 \%$ & $6.20 \%$ & $-0.30 \%$ & $3.00 \%$ \\
\hline
\end{tabular}

\subsubsection{Walking time}

According to the data in Table 3, with the increase of walking time, the passenger score decreases. Passengers can get better walking feeling in the passage with shorter walking time. Further analysis of the declining trend of scores shows that the degree of decline of scores in the $15 \mathrm{~s}-30 \mathrm{~s}$ interval is much less than that in the $30 \mathrm{~s}-45 \mathrm{~s}$ interval, which indicates that passengers have a certain tolerance range for walking time, and the increase of walking time will not cause obvious decline of scores. But beyond this range, passengers' physical strength decline and dissatisfaction with mechanical walking activities will lead to a rapid decline in scores. In addition, the average score between $45 \mathrm{~s}$ and $75 \mathrm{~s}$ is basically the same, which indicates that when the passage length reaches a large value, the time sensitivity of passengers decreases after physical exertion.

Table 3. Descriptive statistics of passage walking time score

\begin{tabular}{|c|c|c|c|c|c|c|}
\hline Walking & $\mathrm{ime} / \mathrm{s}$ & 15 & 30 & 45 & 60 & 75 \\
\hline \multicolumn{2}{|c|}{ Mean score } & 3.08 & 2.97 & 2.43 & 2.28 & 2.38 \\
\hline Confidence & Upper 1 & 3.14 & 3.02 & 2.47 & 2.33 & 2.43 \\
\hline -interval(95\%) & Lower lim & 3.03 & 2.92 & 2.38 & 2.24 & 2.34 \\
\hline \multicolumn{2}{|c|}{ Growth rate of score } & - & $-3.57 \%$ & $-18.18 \%$ & $-6.2 \%$ & $4.3 \%$ \\
\hline
\end{tabular}

\subsubsection{Per capita area}

It can be seen from Table 4 that the score shows an upward trend with the increase of the per capita area. This shows that as the per capita area increases, the chance of passengers colliding with others decreases and the walking experience becomes better. However, the scoring growth rate between each adjacent interval shows a downward trend, which shows that when the per capita area increases to a certain value, the passage space has shown a good walking environment, and passengers' sensitivity to the decrease in the number of people in the passage will decrease.

Table 4. Descriptive statistics of per capita area score

\begin{tabular}{|c|c|c|c|c|c|}
\hline Per capit & $\mathrm{rea} / \mathrm{m}^{2}$ & 1 & 2.5 & 5 & 7.5 \\
\hline \multicolumn{2}{|c|}{ Mean score } & 2.13 & 2.58 & 2.97 & 3.34 \\
\hline \multirow{2}{*}{$\begin{array}{l}\text { Confidence - } \\
\text { interval }(95 \%)\end{array}$} & Upper limit & 2.16 & 2.63 & 3.02 & 3.39 \\
\hline & Lower limit & 2.10 & 2.54 & 2.92 & 3.29 \\
\hline \multicolumn{2}{|c|}{ Growth rate of score } & - & $21.13 \%$ & $15.12 \%$ & $12.46 \%$ \\
\hline
\end{tabular}




\subsubsection{Illumination intensity}

According to the data in Table 5, it can be seen that as the lighting intensity increases, the score shows an upward trend. This shows that the bright environment has a positive effect on the psychology of passengers when they are walking. However, the scoring growth rate of each adjacent interval decreases, which shows that when the illumination intensity in the passage increases to a certain value, the passage has provided a good walking environment, and passengers' sensitivity to the increase of the lighting brightness will decrease.

Table 5. Descriptive statistics of illumination intensity score

\begin{tabular}{|c|c|c|c|c|}
\hline illumination & nsity /lux & 100 & 150 & 200 \\
\hline \multicolumn{2}{|c|}{ Mean score } & 2.38 & 2.75 & 2.88 \\
\hline \multirow{2}{*}{$\begin{array}{l}\text { Confidence - } \\
\text { interval }(95 \%)\end{array}$} & Upper limit & 2.41 & 2.79 & 2.93 \\
\hline & Lower limit & 2.35 & 2.72 & 2.83 \\
\hline \multicolumn{2}{|c|}{ Growth rate of score } & - & $15.55 \%$ & $4.73 \%$ \\
\hline
\end{tabular}

\subsubsection{Guide signage}

The average score of the strip signage is 2.66 , and the average score of the signboard is 2.57 , which means that passengers are more satisfied with the use of the strip signage.

This is due to the large area and continuity of the strip signage, which will make passengers more confident about their position and the target point ahead. Signboard is small in size, and placement interval, poor visual access to information. But both score only a difference of $3 \%$, which shows that although the setting of the signages will affect walking experience, the difference in the perception of passengers isn't very high.

\subsection{Importance ranking analysis}

Use range analysis method to determine the order of the influence of each factor on the score.

Calculate the range of each factor separately, the larger the $R_{j}$, the greater the influence of the corresponding factor on the experimental results. The ranking result of importance is: per capita area $\left(R_{I}=1.21\right)>$ walking time $\left(R_{2}=0.8\right)>\operatorname{width}\left(R_{3}=0.56\right)>$ illumination intensity $\left(R_{4}=0.5\right)>$ guide signage $\left(R_{5}=0.09\right)$.

\section{Passage service level classification}

\subsection{Scoring model establishment}

This section will use stepwise regression to establish a scoring model for the service level of urban rail transit passage, and analyze the Goodness of Fit of the model.

First calibrate the model parameters. Since it is shown in Section 3.2 that the influence of each factor on the score shows obvious positive and negative correlation, and the change of the score shows a linear trend, it's considered to choose the stepwise method for multiple regression analysis to establish a scoring model. Standardize the data, after four iterations of regression, guide signage was excluded from the model, and the significance level of the remaining four factors was less than 0.05 . The scoring model is summarized as Equation 1.

$$
F_{p}=1.23+0.185 a-0.014 t+0.005 b+0.128 w
$$

In Equation 1, $F_{p}$ represents the passenger scoring of the passage service level, $a$ represents the per capita area; $t$ represents the walking time; $b$ represents the illumination intensity; $w$ represents the width of passage.

Use the coefficient of determination $R^{2}$ to perform Goodness of Fit test. $R^{2} \in(0,1)$, the larger the value, the better the fit of the established model. After four iterations of stepwise regression, $R^{2}$ reaches 0.801 , which can better reflect the effect of influencing factors on scoring.

\subsection{Service level number and threshold determination}

Passenger satisfaction refers to how passengers feel about the facilities and operations of urban rail transit. Service level service refers to the quality of the service provided by the operating unit. Passenger satisfaction has a direct relationship with service level: service level determines passenger satisfaction, and passenger satisfaction also reflects the service level of urban rail transit. Therefore, the passenger's satisfaction score for the passage can be transformed into a measure of the service level of the passage in a certain way. The design factors of the passage are connected with the passenger score, and the satisfaction score classification is carried out through data processing, which is transformed into a service level classification.

\subsubsection{Service level number calculation}

The ward connection method is used for clustering, and the metric is run to obtain an approximate matrix of the interval squared Euclidean distance, and the aggregation coefficient is further obtained.

Using the K-means method, on the basis of minimizing the error function, the samples are grouped into $k$ categories according to the similarity of the scoring data, and $k$ is the aggregation number of the $\mathrm{K}$-means. Draw the aggregation coefficient-category number line graph as shown in Fig. 2. When categories $K=4$, the decrease trend of aggregation coefficient slows down, so the $k=4$, that is, the service level is classified as 4 .

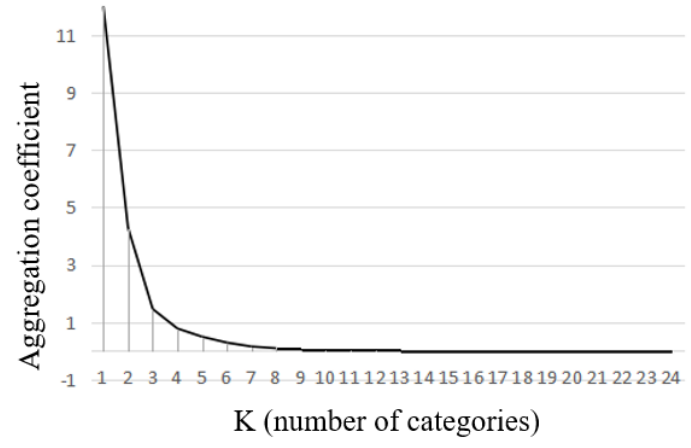

Fig. 2. Aggregation coefficient-category number line graph 


\subsubsection{Classification threshold calculation}

Use MATLAB to program, run and output the clustering results under the K-means method as shown in Table 6. Clustering boundary treatment, complete the connection between the corridor service level and the passenger service level scoring mechanism, as shown in Table 7.

Table 6. Clustering demarcation under K-means method

\begin{tabular}{cccc}
\hline Category & Lower bound & Upper bound & Class center \\
\hline 3.08 & 2.97 & 2.43 & 2.28 \\
3.14 & 3.02 & 2.47 & 2.33 \\
3.03 & 2.92 & 2.38 & 2.24 \\
- & $-3.57 \%$ & $-18.18 \%$ & $-6.2 \%$. \\
\hline
\end{tabular}

Table 7. Service level classification results

\begin{tabular}{cc}
\hline Passage service level & Average passenger evaluation score \\
\hline A & $>3.6$ \\
B & $2.6 \sim 3.6$ \\
C & $2.2 \sim 2.6$ \\
D & $<2.2$ \\
\hline
\end{tabular}

\section{Conclusions}

This paper points out the problems in current researches on the service level of urban rail transit that there are inconsistent evaluation standards, the use of a single evaluation indicator, and the experiments based on passenger-perception cannot independently set up passage scenes based on the researched factors and their levels.

The 5 evaluation indicators for the service level of the passage and their level classifications were determined, 25 experimental scenes were parameterized by orthogonal experiment, and $3 \mathrm{ds}$ max was used to establish these scene models based on the perspective of passengers. The questionnaire was designed and distributed, and a total of 474 valid questionnaires were returned. The reliability coefficient of the questionnaire was 0.935, and the structural validity coefficient was 0.927 . The reliability and validity of the questionnaire were good.

The factor effect analysis results show that the per capita area, width, illumination intensity and the scores are positively correlated, and the passengers' perception sensitivity is reduced when the value exceeds a certain value; the travel time is negatively correlated with the score, and the marginal effect is diminishing; passengers prefer the stripe signage. The importance of the indicators are ranked as follows: per capita area, walking time, width, illumination intensity, and guide signage.

Establish a passenger satisfaction model based on stepwise regression, $R^{2}$ is 0.82 , that is, these indicators can well explain the mechanism of satisfaction scoring. The Kmeans method is used to connect the passenger scoring and the service level of the passage. Finally, the service level of the passage is divided into 4 levels, and the boundary threshold between each level is delineated.

The optimization and innovation of existing research in this paper are as follows. The selection of indicators is comprehensive and realistic. Carry out the passage service level survey from the passenger perspective, and establish the relationship between passenger perception and passage facilities, which takes into account the group nature of passengers and the humanization of services.

This paper can provide a reference for the service level evaluation of existing stations and the design of physical and environmental factors of new stations.

\section{References}

1. Mumayiz S A, Ashford N J. Methodology for planning and operations management of airport terminal facilities[C]. Transportation Research Record, National Research Council, Washington, DC, 1986: 24-35.

2. Anderson R. Correia,S.C. Wirasinghe,Alexandre G. de Barros. Overall level of service measures for airport passenger terminals[J]. Transportation Research Part A,2008,42(2).

3. Shouhua Cao, Zhenzhou Yuan, Chiqing Zhang, Li Zhao. Urban rail transit channel service level division based on passenger perception[J]. Transportation System Engineering and Information,2009,9(02):99104.

4. National Research Council (NRC). Highway capacity manual [R]. Washington, DC: Transportation Research Board, 2000.

5. Kittelson \& Associates, Parsons Brinckerhoff, KFH group, et al. Transit capacity and quality of service manual (the third edition) [R]. Washington, DC: Transportation Research Board, 2013. 